\title{
Boundary effects on electrophoresis of a colloidal cylinder with a nonuniform zeta potential distribution
}

\author{
Tzu H. Hsieh, Huan J. Keh* \\ Department of Chemical Engineering, National Taiwan University, Taipei 10617, Taiwan, Republic of China
}

Received 16 May 2007; accepted 22 June 2007

Available online 31 July 2007

\begin{abstract}
The electrophoretic motion of a long dielectric circular cylinder with a general angular distribution of its surface potential under a transversely imposed electric field in the vicinity of a large plane wall parallel to its axis is analyzed. The thickness of the electric double layers adjacent to the solid surfaces is assumed to be much smaller than the particle radius and the gap width between the surfaces, but the applied electric field can be either perpendicular or parallel to the plane wall. The presence of the confining wall causes three basic effects on the particle velocity: (1) the local electric field on the particle surface is enhanced or reduced by the wall; (2) the wall increases viscous retardation of the moving particle; (3) an electroosmotic flow of the suspending fluid may exist due to the interaction between the charged wall and the tangentially imposed electric field. Through the use of cylindrical bipolar coordinates, the Laplace and Stokes equations are solved analytically for the two-dimensional electric potential and velocity fields, respectively, in the fluid phase, and explicit formulas for the quasisteady electrophoretic and angular velocities of the cylindrical particle are obtained. To apply these formulas, one has only to calculate the multipole moments of the zeta potential distribution at the particle surface. It is found that the existence of a plane wall near a nonuniformly charged particle can cause its translation or rotation which does not occur in an unbounded fluid with the same applied electric field.
\end{abstract}

(c) 2007 Elsevier Inc. All rights reserved.

Keywords: Electrophoresis; Nonuniform zeta potential distribution; Circular cylindrical particle; Plane wall; Boundary effect

\section{Introduction}

A charged particle suspended in an electrolyte solution is surrounded by a diffuse cloud of ions carrying a total charge equal and opposite in sign to that of the particle. This distribution of fixed charge and diffuse ions is known as an electric double layer. When an electric field is imposed on the particle, a force is exerted on both parts of the double layer. The particle is attracted toward the electrode of its opposite sign, while the ions in the diffuse layer migrate in the other direction. This particle motion is termed electrophoresis and has long been applied to the particle characterization or separation in a variety of colloidal and biological systems.

The electrophoretic velocity $\mathbf{U}$ of an isolated particle is related to the applied electric field $\mathbf{E}_{\infty}$ by the Smoluchowski

\footnotetext{
* Corresponding author.

E-mail address: huan@ntu.edu.tw (H.J. Keh).
}

equation [1-3],

$\mathbf{U}=\frac{\varepsilon \zeta_{\mathrm{p}}}{\eta} \mathbf{E}_{\infty}$

Here, $\eta$ and $\varepsilon$ represent the viscosity and permittivity, respectively, of the solution surrounding the particle, and $\zeta_{\mathrm{p}}$ is the zeta potential associated with the particle surface. This formula is valid on the basis of several assumptions: (i) the local radii of curvature of the particle are much larger than the thickness of its electric double layer; (ii) the ambient fluid is unbounded; (iii) the zeta potential is uniform on the length scale of the particle. The first restriction also implies that the double layer remains approximately in equilibrium despite the migration of the particle and diffuse ions. Even though many colloidal particles undergoing electrophoresis fulfill this condition, electrophoresis of particles with thick or distorted double layers does occur in certain cases so that relevant corrections to the Smoluchowski prediction in Eq. (1) are necessary and have been obtained [4-8]. 
In practical applications of electrophoresis, colloidal particles are not isolated and will move in the presence of neighboring boundaries [9-11]. Therefore, the boundary effects on electrophoresis are of great importance and have been studied extensively in the past for various cases of uniformly charged colloidal spheres and boundaries in the limit of thin electric double layers. Using a method of reflections, Keh and Anderson [12] analyzed the electrophoretic motions of a dielectric sphere normal to a large conducting plane, parallel to a large dielectric plane, along the axis of a long circular tube, and along the central plane between two large parallel plates. Through an exact representation in spherical bipolar coordinates or a lubrication theory, semianalytical solutions for the electrophoretic velocity of a colloidal sphere in the vicinity of an infinite plane wall have also been obtained in two principal cases: the migration perpendicular to a conducting plane [13-15] and the movement parallel to an insulating wall [16,17]. Subsequently, the boundary effects on electrophoresis of a charged sphere were investigated for geometries like migration along the axis of a circular orifice or disk [18], movement in a circular cylindrical pore at an axial [19] or eccentric [20,21] position, and motion in between two parallel plane walls [22-24]. The boundary effects on electrophoresis have also been theoretically examined for the cases of spherical particles with thick or distorted double layers [24-28] and of nonspherical particles [29-31].

On the other hand, many colloidal particles have heterogeneous surface structures or chemistry and are nonuniformly charged. For instance, elementary clay particles are flat disks with edges having a different charge density or zeta potential from the faces. Distributions of surface charge or potential for particles can also result from aggregation of different species of colloids. Even if a particle is homogeneously charged on its surface, an applied electric field could cause rearrangement of these charges if they are mobile [32]. A distribution of zeta potential on particle surfaces has been found to lead to colloidal instability, even the average zeta potential should be sufficiently high to keep the suspension stable $[33,34]$. The electrophoretic motion of a dielectric sphere with nonuniform zeta potential and thin electric double layer was first analyzed thoroughly by Anderson [35], although it had also been discussed to some extent earlier [36]. It was found that, in terms of the multipole moments of the zeta potential, the electrophoretic mobility depends not only on the monopole moment (area-averaged zeta potential) but also on the quadrupole moment, and the dipole moment contributes to particle rotation which tends to align the particle with the electric field. This analysis was later extended to the cases of a nonuniformly charged spherical particle with a double layer of finite thickness [37-40] and a nonuniformly charged nonspherical particle [41-45]. Recently, that particles can have random charge nonuniformity has also been demonstrated experimentally $[46,47]$.

The electrophoretic motion of nonuniformly charged particles in the proximity of confining walls could also be encountered in some real situations. For example, the translation and rotation of each of an array of nonuniformly charged bichromal spheres in its own elastomer-made and solvent-filled cavity controlled by imposing a voltage of either positive or negative polarity have been applied to a technology of electric paper displays $[48,49]$. Also, an electrophoretic positioning process has been employed in electronic applications for assembling very small individual devices, such as an InGaAs light-emitting diode or a nanowire, which is nonuniformly charged and must have all electric contacts available on one surface, onto the contact electrodes of a silicon circuit by biasing the contacts to control the placement of these devices with the precision required [50,51]. Recently, the electrophoresis of a dielectric spherical particle in a concentric spherical cavity with nonuniform zeta potential distributions at the solid surfaces has been investigated and analytical expressions for the translational and angular velocities of the particle in terms of the monopole, dipole, and quadrupole moments of the zeta potentials were obtained [52].

The objective of this paper is to determine the electrophoretic velocity of a long dielectric circular cylinder with an a nonuniform zeta potential distribution in the angular direction near a large plane wall parallel to its axis in transversely applied electric fields. The electric double layers are assumed to be thin compared with the radius of the cylindrical particle and with the surface-to-surface spacing between the particle and the wall. A cylindrical bipolar coordinate system is used to solve the quasisteady problem. In the next section, the electrophoresis of a circular cylinder caused by an imposed electric field in the direction perpendicular to its axis and to a conducting plane wall is examined. The analytical solution for the wall-corrected electrophoretic velocity of the particle is obtained in Eqs. (20a) and (20b). The analysis of a complementary problem to that treated in Section 2, the electrophoretic motion of a circular cylinder driven by an applied electric field in the direction perpendicular to its axis and parallel to a dielectric plane wall, is presented in Section 3. The general expressions for the electrophoretic velocity of the particle in this case are given in Eqs. (28a)-(28c).

\section{Electrophoresis in an applied electric field perpendicular to a conducting plane wall}

In this section we consider the quasisteady electrophoretic motion of a long circular cylindrical particle of radius $a$ caused by a uniform electric field $\mathbf{E}_{\infty}=E_{\infty} \mathbf{e}_{x}$ imposed normal to its axis and to a large conducting plane wall located at a distance $d$ from the axis, as illustrated in Fig. 1a, where $\mathbf{e}_{x}$ together with $\mathbf{e}_{y}$ and $\mathbf{e}_{z}$ are the principal unit vectors in the Cartesian coordinate system $(x, y, z)$ with a right-handed screw. The zeta potential $\zeta_{\mathrm{p}}$ on the surface of the particle at $r=a$ can be a general function of the azimuth angle $\theta$, where $(r, \theta, z)$ are circular cylindrical coordinates. The thickness of the electric double layers surrounding the particle and adjacent to the plane wall is assumed to be much smaller than the radius of the cylinder and the spacing between the solid surfaces. Gravitational and end effects are neglected. Our purpose is to determine the electrophoretic velocity of the nonuniformly charged cylindrical particle in the presence of the plane wall.

For convenience in satisfying the boundary conditions at the solid surfaces, an orthogonal curvilinear coordinate system $(\xi, \psi, z)$ known as cylindrical bipolar coordinates and shown in Fig. 2 is utilized to solve the problem. This coordinate system 


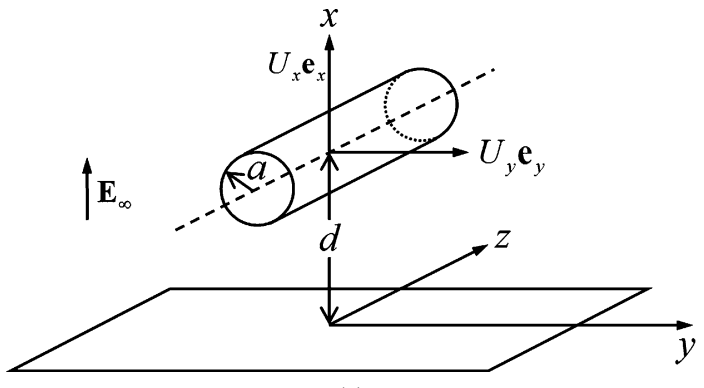

(a)

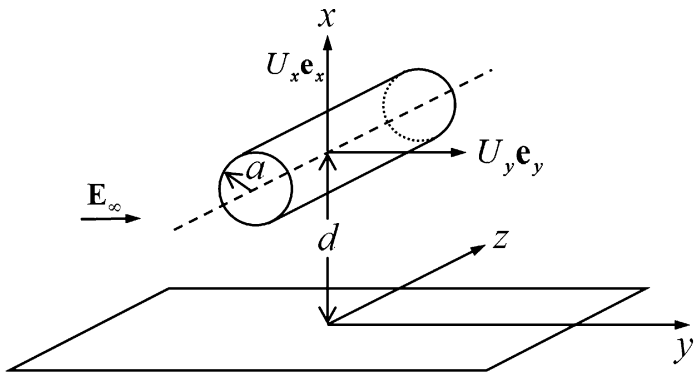

(b)

Fig. 1. Geometric sketch for the transverse electrophoresis of a circular cylinder in the proximity of a plane wall: (a) electric field applied perpendicular to the wall; (b) electric field imposed parallel to the wall.

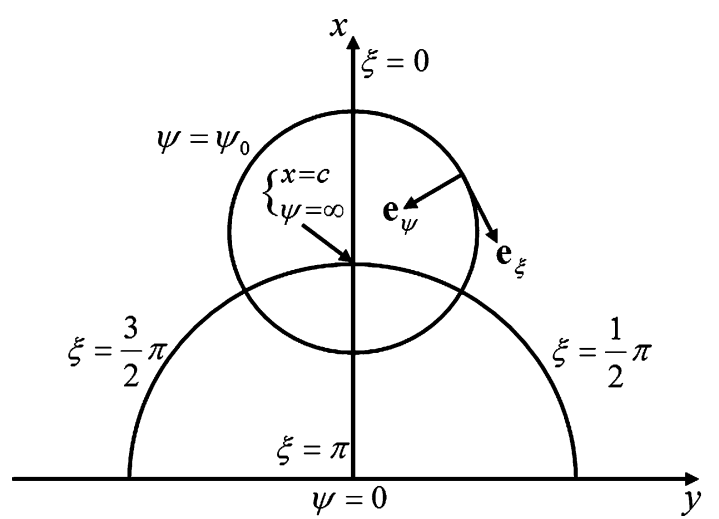

Fig. 2. The two-dimensional bipolar coordinates $(\xi, \psi)$ and rectangular coordinates $(x, y)$.

is related to rectangular coordinates in any plane $z=$ constant by the relation $[53,54]$

$x=\frac{c \sinh \psi}{\cosh \psi-\cos \xi}$,

$y=\frac{c \sin \xi}{\cosh \psi-\cos \xi}$,

where $-\infty<\psi<\infty, 0 \leqslant \xi \leqslant 2 \pi$, and $c$ is a characteristic length in the bipolar coordinate system which is positive.

The curves $\psi=$ constant correspond to a family of nonintersecting, coaxial circles (or cylinders) whose centers all lie along the $x$ axis. The special case $\psi=0$ generates a circle of infinite radius and corresponds to the entire $y$ axis (or the plane $x=0$ ). $\psi=\psi_{0}>0$ represents the circle (or the cylinder) of radius $a=c \operatorname{csch} \psi_{0}$, with its center at the point $\left(x=d=c \operatorname{coth} \psi_{0}\right.$, $y=0$ ). The ratio of the radius of the cylinder to the distance of the axis of the cylinder from the plane is related to $\psi_{0}$ by

$\lambda=a / d=\operatorname{sech} \psi_{0}$.

Before determining the electrophoretic velocity of the cylindrical particle near the plane wall, the electric potential and velocity fields in the fluid phase must be solved.

\subsection{Electric potential distribution}

The fluid outside the thin double layers is electrically neutral and of constant conductivity; hence the electric potential distribution $\Phi(\xi, \psi)$ is governed by the Laplace equation,

$\nabla^{2} \Phi=0$

Here, the operator $\nabla^{2}$ in bipolar coordinates has the form

$\nabla^{2}=\frac{1}{c^{2}}(\cosh \psi-\cos \xi)^{2}\left(\frac{\partial^{2}}{\partial \xi^{2}}+\frac{\partial^{2}}{\partial \psi^{2}}\right)$.

The potential gradient far away from the cylinder approaches the applied electric field, and the cylindrical particle is assumed to be perfectly insulating. Also, the plane boundary is considered as a perfectly conducting wall and its potential is taken to be zero for convenience. Thus, the boundary conditions for $\Phi$ are

$\psi=\psi_{0}: \quad \mathbf{e}_{\psi} \cdot \nabla \Phi=0$,

$\psi=0: \quad \Phi \rightarrow-E_{\infty} x$,

where

$\nabla=\frac{1}{c}(\cosh \psi-\cos \xi)\left(\mathbf{e}_{\xi} \frac{\partial}{\partial \xi}+\mathbf{e}_{\psi} \frac{\partial}{\partial \psi}\right)$,

and $\mathbf{e}_{\xi}$ and $\mathbf{e}_{\psi}$ are the principal unit vectors in bipolar coordinates. Note that

$\mathbf{e}_{x}=\frac{1}{\cosh \psi-\cos \xi}\left[-\sinh \psi \sin \xi \mathbf{e}_{\xi}\right.$

$$
\left.-(\cosh \psi \cos \xi-1) \mathbf{e}_{\psi}\right] \text {, }
$$

$\mathbf{e}_{y}=\frac{1}{\cosh \psi-\cos \xi}\left[(\cosh \psi \cos \xi-1) \mathbf{e}_{\xi}-\sinh \psi \sin \xi \mathbf{e}_{\psi}\right]$.

The solution to Eq. (4) subject to the boundary conditions in Eqs. (6a) and (6b) is [29]

$$
\begin{aligned}
\Phi= & -2 c E_{\infty} \sum_{n=1}^{\infty} e^{-n \psi_{0}} \operatorname{sech} n \psi_{0} \sinh n \psi \cos n \xi \\
& -c E_{\infty} \frac{\sinh \psi}{\cosh \psi-\cos \xi},
\end{aligned}
$$

in which the last term is the electric potential distribution that would exist in the absence of the cylinder.

\subsection{Fluid velocity distribution}

With knowledge of the solution for the electric potential distribution in the fluid phase, we can now proceed to find the fluid flow field. Because the Reynolds number associated with electrophoretic motions is small, the velocity distribution for the 
fluid outside the thin electric double layers is governed by the Stokes equations,

$\eta \nabla^{2} \mathbf{v}-\nabla p=\mathbf{0}$,

$\nabla \cdot \mathbf{v}=0$

where $\mathbf{v}$ is the fluid velocity distribution and $p$ is the dynamic pressure. Taking the curl of both sides of Eq. (10a) and introducing Eq. (10b) and the stream function $\Psi$ result in a fourthorder linear partial differential equation,

$\nabla^{4} \Psi=\nabla^{2}\left(\nabla^{2} \Psi\right)=0$

The stream function is related to the velocity components in bipolar coordinates by the formulas

$v_{\xi}=\frac{1}{c}(\cosh \psi-\cos \xi) \frac{\partial \Psi}{\partial \psi}$,

$v_{\psi}=-\frac{1}{c}(\cosh \psi-\cos \xi) \frac{\partial \Psi}{\partial \xi}$.

At the surface of the cylinder, the electric field acting on the diffuse ions within the double layer produces a relative tangential fluid velocity at the outer boundary of the double layer as given by the Helmholtz expression for the electroosmotic flow [55]. At a distance far away from the particle and on the conducting plane wall, the fluid is motionless. Therefore, the boundary conditions for the velocity field are

$\psi=\psi_{0}: \quad \mathbf{v}=U_{x} \mathbf{e}_{x}+U_{y} \mathbf{e}_{y}+a \Omega \mathbf{e}_{\xi}+\frac{\varepsilon \zeta_{\mathrm{p}}}{\eta} \nabla \Phi$,

$\psi=0: \quad \mathbf{v} \rightarrow \mathbf{0}$,

where $U_{x} \mathbf{e}_{x}+U_{y} \mathbf{e}_{y}$ and $\Omega \mathbf{e}_{z}$ are the translational and angular velocities, respectively, of the electrophoretic cylinder to be determined and the expression for $\Phi$ is given by Eq. (9). Note that $U_{y}$ and $\Omega$ appear in Eq. (13a) since the zeta potential $\zeta_{\mathrm{p}}$ can be a general function of the angular position on the particle surface. Because the cylinder is freely suspended in the fluid, the net force and net torque exerted by the fluid on the cylinder per unit length must vanish.

Since the governing equation and boundary conditions are linear, the total flow can be decomposed into two parts. First, we consider the fluid velocity field $\mathbf{v}_{1}$ about a circular cylinder (with its surface at $\psi=\psi_{0}$ ) translating with a velocity $U_{x} \mathbf{e}_{x}+$ $U_{y} \mathbf{e}_{y}$ and rotating with an angular velocity $\Omega \mathbf{e}_{z}$ near a plane wall (at $x=0$ ), but with no electrokinetic slip velocity at the particle surface. The stream function for this creeping flow was obtained and the drag force $\mathbf{F}_{1}$ and torque $\mathbf{T}_{1}$ exerted by the fluid on the cylinder per unit length is [29]

$\mathbf{F}_{1}=-4 \pi \eta\left(\frac{U_{x}}{\psi_{0}-\tanh \psi_{0}} \mathbf{e}_{x}+\frac{U_{y}}{\psi_{0}} \mathbf{e}_{y}\right)$,

$\mathbf{T}_{1}=-4 \pi \eta a^{2} \Omega \operatorname{coth} \psi_{0} \mathbf{e}_{z}$.

The above equations indicate that the translation and rotation for the two-dimensional creeping motion of a circular cylinder near a plane wall are not coupled with each other.

Next, we consider the fluid flow caused by the electrokinetic tangential velocity at the surface (outer edge of the electric double layer) of a stationary circular cylinder near a plane wall, namely, the flow subject to the boundary conditions given by Eqs. (13a) and (13b) with $U_{x}=U_{y}=\Omega=0$. Superposing this velocity field $\mathbf{v}_{2}$ with $\mathbf{v}_{1}$ will yield the total velocity field produced by the electrophoretic motion of a cylinder under an applied electric field normal to its axis and to a plane wall. By obtaining the hydrodynamic force $\mathbf{F}_{2}$ and torque $\mathbf{T}_{2}$ exerted on the stationary cylinder, adding them respectively to the force $\mathbf{F}_{1}$ and torque $\mathbf{T}_{1}$ given by Eqs. (14a) and (14b), and equating the sums to zero, the translational and angular velocities of the electrophoretic cylinder with wall corrections will result.

The zeta potential $\zeta_{\mathrm{p}}$ is a general function of the azimuth angle $\theta$ on the cylinder surface $r=a$ and can be expressed in terms of the multipole expansions,

$\zeta_{\mathrm{p}}=M+\mathbf{D} \cdot \mathbf{e}_{r}+\mathbf{Q}: \mathbf{e}_{r} \mathbf{e}_{r}$.

Here $\mathbf{e}_{r}$ and $\mathbf{e}_{\theta}$ are the basic unit vectors in polar coordinates $(r, \theta)$, and the monopole, dipole, and quadrupole moments $M$, $\mathbf{D}$, and $\mathbf{Q}$, respectively, are defined by the following integrals over the particle surface,

$M=\frac{1}{2 \pi} \int_{0}^{2 \pi} \zeta_{\mathrm{p}} \mathrm{d} \theta$,

$\mathbf{D}=\frac{1}{\pi} \int_{0}^{2 \pi} \zeta_{\mathrm{p}} \mathbf{e}_{r} \mathrm{~d} \theta$,

$\mathbf{Q}=\frac{1}{\pi} \int_{0}^{2 \pi} \zeta_{\mathrm{p}}\left(\mathbf{e}_{r} \mathbf{e}_{r}-\mathbf{e}_{\theta} \mathbf{e}_{\theta}\right) \mathrm{d} \theta$,

and the higher-order moments are neglected. Various distributions of nonuniform zeta potential $\zeta_{\mathrm{p}}$ can result from appropriate choices of the moments $M$ (i.e., area-averaged zeta potential), $\mathbf{D}$, and $\mathbf{Q}$ (which is symmetric and traceless).

A general solution to the biharmonic equation (11) in bipolar coordinates, suitable for satisfying boundary conditions on the cylindrical particle and plane wall, has been given by $[53,56]$

$$
\begin{aligned}
\Psi= & \frac{\varepsilon E_{\infty} c}{\eta}(\cosh \psi-\cos \xi)^{-1}[A \psi(\cosh \psi-\cos \xi) \\
& +(B+C \psi) \sinh \psi-D \psi \sin \xi \\
& +\sum_{n=1}^{\infty}\left\{\left[a_{n} \cosh (n+1) \psi+b_{n} \sinh (n+1) \psi\right.\right. \\
& \left.+c_{n} \cosh (n-1) \psi+d_{n} \sinh (n-1) \psi\right] \cos n \xi \\
& +\left[a_{n}^{\prime} \cosh (n+1) \psi+b_{n}^{\prime} \sinh (n+1) \psi\right. \\
& \left.\left.\left.+c_{n}^{\prime} \cosh (n-1) \psi+d_{n}^{\prime} \sinh (n-1) \psi\right] \sin n \xi\right\}\right]
\end{aligned}
$$

The coefficients $A, B, C, D, a_{n}, b_{n}, c_{n}, d_{n}, a_{n}^{\prime}, b_{n}^{\prime}, c_{n}^{\prime}$, and $d_{n}^{\prime}$ (in which $d_{1}$ and $d_{1}^{\prime}$ are trivial) should be determined by the boundary conditions given by Eqs. (13a) and (13b) with $U_{x}=U_{y}=\Omega=0$ using Eqs. (9), (12a), (12b), and (15). After considerable algebraic manipulation, analytical results of these coefficients are obtained and given in Appendix A. 
The drag force and torque exerted on the stationary cylinder per unit length by the fluid due to the electrokinetic motion are

$\mathbf{F}_{2}=4 \pi \varepsilon E_{\infty}\left(D \mathbf{e}_{x}+C \mathbf{e}_{y}\right)$,

$\mathbf{T}_{2}=-4 \pi \varepsilon E_{\infty} a\left(A \sinh \psi_{0}+C \cosh \psi_{0}\right) \mathbf{e}_{z}$,

where the coefficients $A, C$, and $D$ are given by Eqs. (A.1), (A.3), and (A.4).

\subsection{Derivation of the particle velocities}

Since the net hydrodynamic force and torque acting on the electrophoretic cylinder must vanish, we have

$\mathbf{F}_{1}+\mathbf{F}_{2}=\mathbf{0}$,

$\mathbf{T}_{1}+\mathbf{T}_{2}=\mathbf{0}$.

With the substitution of Eqs. (14a), (14b) and (18a), (18b) into the above constraints, the translational velocities $U_{x}$ and $U_{y}$ as well as the angular velocity $\Omega$ of the cylinder near the conducting plane wall are determined as

$$
\begin{aligned}
U_{x}= & \frac{\varepsilon E_{\infty}}{\eta} \sinh \psi_{0} \tanh \psi_{0} \operatorname{sech} 2 \psi_{0}\left\{2 M \sinh \psi_{0}-D_{x} \tanh \psi_{0}\right. \\
& +\frac{1}{4} Q_{x x}\left[\left(\cosh 4 \psi_{0}+1\right) O_{1}+\cosh 2 \psi_{0}\left(R_{1}-4 O_{1}\right)\right. \\
& \left.\left.+6 \sinh \psi_{0}-2 \sinh 3 \psi_{0}\right]\right\} \\
U_{y}= & \frac{\varepsilon E_{\infty}}{\eta} \tanh \psi_{0} \operatorname{sech} 2 \psi_{0}\left\{-D_{y} \cosh \psi_{0}\right. \\
& \left.+\frac{1}{4} Q_{y x}\left[6+2 \cosh 4 \psi_{0}-\left(\sinh 3 \psi_{0}+\sinh 5 \psi_{0}\right) P_{0}\right]\right\}
\end{aligned}
$$

$$
\begin{aligned}
\Omega= & \frac{\varepsilon E_{\infty}}{\eta a}\left\{-D_{y} \tanh 2 \psi_{0}+2 Q_{x y}\left[\left(1-\cosh 2 \psi_{0}\right) P_{0}\right.\right. \\
& \left.\left.+\left(2+\operatorname{sech} 2 \psi_{0}\right) \sinh \psi_{0}-\operatorname{sech} \psi_{0} \tanh \psi_{0}\right]\right\},
\end{aligned}
$$

where $O_{n}, P_{n}$, and $R_{n}$ are defined by Eqs. (A.21)-(A.24).

In the limit $\lambda \rightarrow 0$, Eqs. (20a)-(20c) reduce to

$$
\begin{aligned}
& \mathbf{U}_{0}=\frac{\varepsilon}{\eta}\left(M \mathbf{I}-\frac{1}{2} \mathbf{Q}\right) \cdot \mathbf{E}_{\infty}, \\
& \boldsymbol{\Omega}_{0}=\frac{\varepsilon}{\eta a} \mathbf{D} \times \mathbf{E}_{\infty},
\end{aligned}
$$

which are the translational and angular velocities of a nonuniformly charged circular cylinder undergoing two-dimensional electrophoresis in an unbounded fluid.

For a uniformly charged circular cylinder undergoing electrophoretic motion in a transversely applied electric field normal to a conducting plane wall, Eqs. (20a)-(20c) become

$U_{x}=\frac{2 \varepsilon \zeta_{\mathrm{p}} E_{\infty}}{\eta} \frac{\sinh ^{2} \psi_{0}}{\cosh 2 \psi_{0}} \tanh \psi_{0}$,

and $U_{y}=\Omega=0$. Equation (22) corrects an inadvertent error for the previous result obtained by Keh et al. [29] in their Eq. (4.10).

\subsection{Results and discussion}

The analytical solution for the translational and angular velocities of the cylindrical particle undergoing transverse electrophoresis under the applied electric field $\mathbf{E}_{\infty}=E_{\infty} \mathbf{e}_{x}$ perpendicular to a conducting plane wall is obtained in Eqs. (20a)(20c). For illustrative examples, we consider four cases of the zeta potential distribution on the surface of the particle.

Case I: $\quad \zeta_{\mathrm{p}}=\zeta_{0} \sin \theta$,

Case II: $\quad \zeta_{\mathrm{p}}=\zeta_{0} \cos \theta$,

Case III: $\quad \zeta_{\mathrm{p}}=\zeta_{0} \sin 2 \theta$,

Case IV: $\quad \zeta_{\mathrm{p}}=\zeta_{0} \cos 2 \theta$,

where $\zeta_{0}$ is a constant and $\theta$ is the azimuth angle clockwise from the positive $x$ axis in Fig. 2. Note that both the monopole moment (area-averaged zeta potential) and the quadrupole moment disappear in Cases I and II, while both the monopole and the dipole moments vanish in Cases III and IV. After calculating the multipole moments according to Eqs. (16a)-(16c) and substituting them into Eqs. (20a)-(20c), we obtain the translational and angular velocities of the cylindrical particle for each of the four cases as functions of $\lambda$, the ratio of the radius of the cylinder to the distance of the axis of the cylinder from the plane wall.

For Case I, the dipole moment $\mathbf{D}=\zeta_{0} \mathbf{e}_{y}$, and there is no particle velocity in the direction of the imposed electric field $\left(U_{x}=0\right)$ owing to the antisymmetry of the zeta potential distribution on the surface of the cylindrical particle about the $x$ axis (or the plane $y=0$ ), regardless of the value of the parameter $\lambda$. The results of the lateral velocity $U_{y}$ and angular velocity $\Omega$ of the particle as functions of $\lambda$ are plotted in Fig. 3. The existence of the conducting plane wall depresses the local electric field at the particle surface on the side next to the wall compared with that on the far side [29], and thus reduces the magnitude of the angular velocity of the particle from $\varepsilon\left|\zeta_{0}\right| E_{\infty} / \eta a$ (in the direction of $-\mathbf{e}_{z} \zeta_{0} /\left|\zeta_{0}\right|$, as predicted by Eq. (21b)) at $\lambda=0$ to zero at

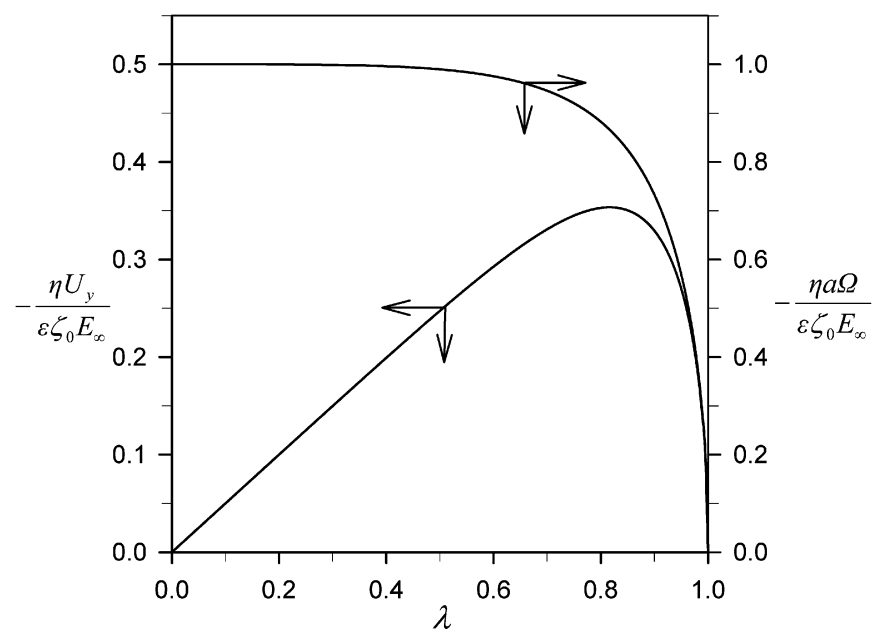

Fig. 3. Plots of the normalized velocities $-\eta U_{y} / \varepsilon \zeta_{0} E_{\infty}$ and $-\eta a \Omega / \varepsilon \zeta_{0} E_{\infty}$ of a circular cylinder with a zeta potential distribution given by Eq. (23a) in a transversely applied electric field perpendicular to a conducting plane wall versus the separation parameter $\lambda$. 


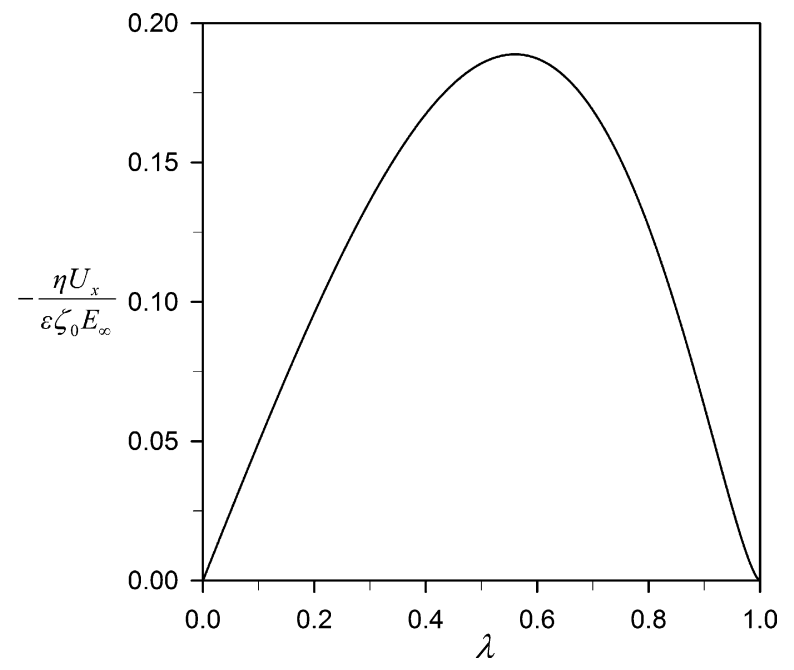

Fig. 4. Plots of the normalized velocity $-\eta U_{x} / \varepsilon \zeta_{0} E_{\infty}$ of a circular cylinder with a zeta potential distribution given by Eq. (23b) in a transversely applied electric field perpendicular to a conducting plane wall versus the separation parameter $\lambda$.

$\lambda=1$. Interestingly, there exists an accompanying finite lateral velocity $U_{y}$ of the cylinder in the direction of $-\mathbf{e}_{y} \zeta_{0} /\left|\zeta_{0}\right|$ (predictable from observing the strength and direction distributions of the tangential electroosmotic velocity at the particle surface given in Eq. (13a)) as long as $0<\lambda<1$, but this velocity disappears in both limits of $\lambda$ as expected. It can be shown that the maximal magnitude of $U_{y}$ equals $\varepsilon\left|\zeta_{0}\right| E_{\infty} / 2 \sqrt{2} \eta$, which occurs at $\lambda=\sqrt{2 / 3}$.

For Case II defined by Eq. (23b), the dipole moment $\mathbf{D}=$ $\zeta_{0} \mathbf{e}_{x}$, and $U_{y}=\Omega=0$ due to the symmetry of the zeta potential distribution on the surface of the cylindrical particle about the $x$ axis, irrespective of the value of $\lambda$. As shown in Fig. 4, the electrophoretic velocity of the particle, $U_{x}$, has a finite magnitude in the direction of $-\mathbf{e}_{x} \zeta_{0} /\left|\zeta_{0}\right|$ for all finite separations $(0<\lambda<1)$ and, as expected, vanishes in both limits of $\lambda$. It can be found that the maximal magnitude of $U_{x}$ equals $(3+\sqrt{33})^{3 / 2} \varepsilon\left|\zeta_{0}\right| E_{\infty} / 4(17+3 \sqrt{33}) \eta$, which takes place at $\lambda=2 /(7+\sqrt{33})^{1 / 2}$.

For Case III, the quadrupole moment $\mathbf{Q}=\zeta_{0}\left(\mathbf{e}_{x} \mathbf{e}_{y}+\mathbf{e}_{y} \mathbf{e}_{x}\right)$, and there is no velocity of the cylinder in the direction of the applied electric field $\left(U_{x}=0\right)$ for any value of $\lambda$, owing to the antisymmetry of the zeta potential distribution on the particle surface about the $x$ axis. The results of the lateral velocity $U_{y}$ and angular velocity $\Omega$ of the particle as functions of $\lambda$ are plotted in Fig. 5. For an isolated cylinder (with $\lambda=0$ ), the particle translates with a lateral velocity $U_{y}=-\varepsilon \zeta_{0} E_{\infty} / \eta$ without rotation, as given by Eqs. (21a)-(21b). The existence of the conducting plane wall (with a finite value of $\lambda$ ) reduces the magnitude of this lateral velocity and causes a finite angular velocity $\Omega$ of the cylinder in the direction of $\mathbf{e}_{z} \zeta_{0} /\left|\zeta_{0}\right|$, and both velocities vanish in the limit $\lambda=1$. The maximal magnitude of $\Omega$ equals about $0.176 \varepsilon\left|\zeta_{0}\right| E_{\infty} / \eta a$, which occurs near $\lambda=0.915$.

For Case IV defined by Eq. (23d), the quadrupole moment $\mathbf{Q}=\zeta_{0}\left(\mathbf{e}_{x} \mathbf{e}_{x}-\mathbf{e}_{y} \mathbf{e}_{y}\right)$, and $U_{y}=\Omega=0$ for any value of $\lambda$ due to the symmetry of the zeta potential distribution on the parti-

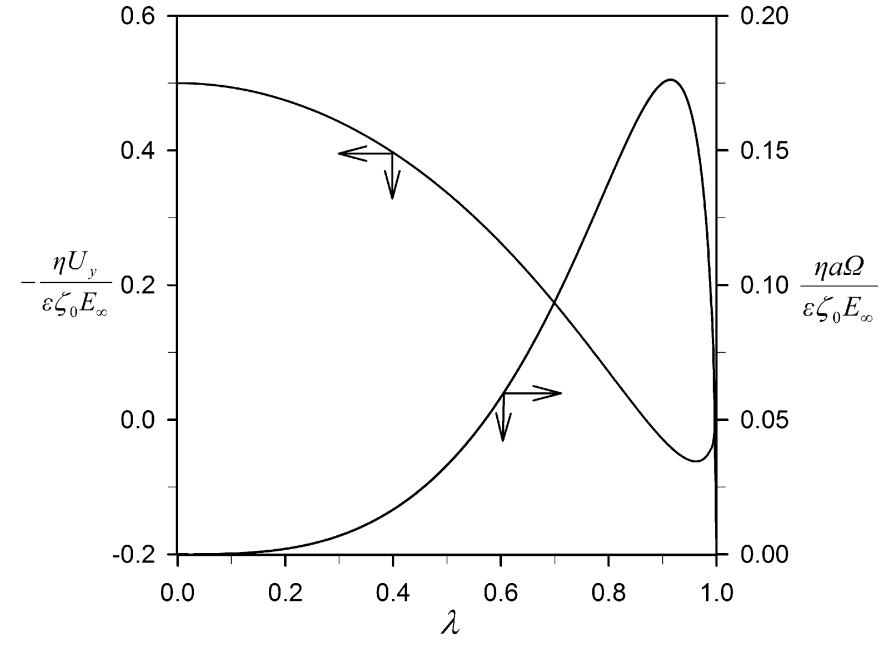

Fig. 5. Plots of the normalized velocities $-\eta U_{y} / \varepsilon \zeta_{0} E_{\infty}$ and $\eta a \Omega / \varepsilon \zeta_{0} E_{\infty}$ of a circular cylinder with a zeta potential distribution given by Eq. (23c) in a transversely applied electric field perpendicular to a conducting plane wall versus the separation parameter $\lambda$.

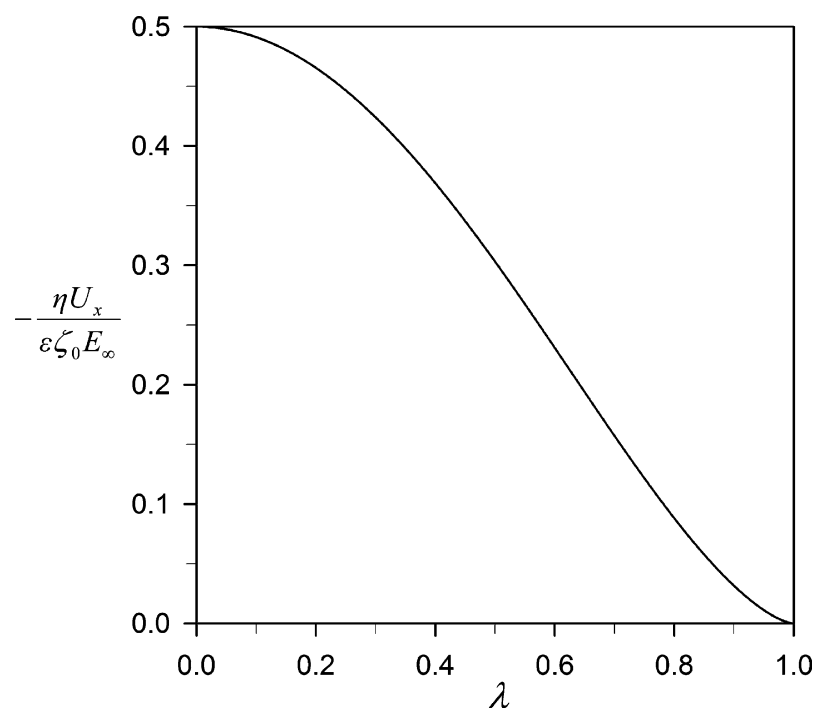

Fig. 6. Plots of the normalized velocity $-\eta U_{x} / \varepsilon \zeta_{0} E_{\infty}$ of a circular cylinder with a zeta potential distribution given by Eq. (23d) in a transversely applied electric field perpendicular to a conducting plane wall versus the separation parameter $\lambda$.

cle surface about the $x$ axis. The result of the particle velocity in the direction of the imposed electric field, $U_{x}$, as a function of $\lambda$ is plotted in Fig. 6. For the case of an unconfined cylinder (with $\lambda=0$ ), the particle translates with an electrophoretic velocity $U_{x}=-\varepsilon \zeta_{0} E_{\infty} / 2 \eta$, as predicted by Eq. (21a). When the conducting plane wall exists, as expected, the magnitude of this electrophoretic velocity decreases with an increase in $\lambda$ and vanishes in the limit $\lambda=1$.

\section{Electrophoresis in an applied electric field parallel to an insulating plane wall}

We now consider the two-dimensional quasisteady electrophoretic motion of a long circular cylinder of radius $a$ (repre- 
sented by $\psi=\psi_{0}$ ) under a uniform electric field $\mathbf{E}_{\infty}=E_{\infty} \mathbf{e}_{y}$ imposed perpendicular to its axis (with coordinates $x=d$ and $y=0$ ) and parallel to a large dielectric plane wall (located at $x=0$ ), as shown in Fig. 1b. The zeta potential distribution on the surface of the cylindrical particle can be a general function of the azimuth angle $\theta$. As in the previous section, the assumption of thin electric double layers is employed. Our objective is to find the wall-corrected electrophoretic velocity of the particle.

\subsection{Electric potential distribution}

The electrostatic equation governing the potential distribution $\Phi(\xi, \psi)$ is the Laplace equation (4). Since the potential gradient far away from the cylinder approaches the applied electric field and both the cylinder and the wall are perfectly insulating, the electric potential is subject to the boundary conditions

$x=0: \quad \mathbf{e}_{\psi} \cdot \nabla \Phi=0$,

$\psi=\psi_{0}: \quad \mathbf{e}_{\psi} \cdot \nabla \Phi=0$,

$\left(x^{2}+y^{2}\right)^{1 / 2} \rightarrow \infty \quad$ and $\quad x>0: \quad \Phi \rightarrow-E_{\infty} y$.

The solution of Eq. (4) satisfying the above boundary conditions is given by [29]

$$
\begin{aligned}
\Phi= & -2 c E_{\infty} \sum_{n=1}^{\infty} e^{-n \psi_{0}} \operatorname{csch} n \psi_{0} \cosh n \psi \sin n \xi \\
& -c E_{\infty} \frac{\sin \xi}{\cosh \psi-\cos \xi},
\end{aligned}
$$

where the last term is the undisturbed potential distribution (in the absence of the particle).

\subsection{Fluid velocity distribution}

Having obtained the solution for the electric potential distribution in the fluid phase, we can now proceed to find the flow field. The fluid motion outside the thin electric double layers is governed by Eq. (11) and subject to the boundary conditions

$$
\begin{aligned}
& x=0: \quad \mathbf{v}=\frac{\varepsilon \zeta_{\mathrm{w}}}{\eta} \nabla \Phi, \\
& \psi=\psi_{0}: \quad \mathbf{v}=U_{x} \mathbf{e}_{x}+U_{y} \mathbf{e}_{y}+a \Omega \mathbf{e}_{\xi}+\frac{\varepsilon \zeta_{\mathrm{p}}}{\eta} \nabla \Phi, \\
& \left(x^{2}+y^{2}\right)^{1 / 2} \rightarrow \infty \quad \text { and } \quad x>0: \\
& \mathbf{v} \rightarrow v_{\infty} \mathbf{e}_{y}=-\frac{\varepsilon \zeta_{\mathrm{w}} E_{\infty}}{\eta} \mathbf{e}_{y},
\end{aligned}
$$

where $\zeta_{\mathrm{w}}$ is the zeta potential associated with the plane wall, which is taken as a constant, $U_{x} \mathbf{e}_{x}+U_{y} \mathbf{e}_{y}$ and $\Omega \mathbf{e}_{z}$ are the translational and angular velocities, respectively, of the electrophoretic cylinder to be determined, and the expression for $\Phi$ is given by Eq. (25). Note that $U_{x}$ exists in Eq. (26b) due to the nonuniformity of $\zeta_{p}$ on the particle surface, and Eqs. (26a) and (26c) allow an electroosmotic flow induced by the interaction of the applied electric field with the charged plane wall.
Similarly to the case dealt with in the previous section, the total flow can be decomposed into two parts. First, we consider the fluid velocity field $\mathbf{v}_{1}$ about a circular cylinder moving near the plane wall with the translational velocity $U_{x} \mathbf{e}_{x}+U_{y} \mathbf{e}_{y}$ and angular velocity $\Omega \mathbf{e}_{z}$, while the plane wall and the fluid far away from the cylinder are moving with a velocity equal to $v_{\infty} \mathbf{e}_{y}$, but with no electrokinetic slip velocity at either of the solid surfaces. For this creeping flow, the drag force $\mathbf{F}_{1}$ and torque $\mathbf{T}_{1}$ per unit length exerted by the fluid on the cylinder is still given by Eqs. (14a), (14b) with $U_{y}-v_{\infty}$ to replace $U_{y}$.

Next, we consider the fluid flow caused by the electrokinetic tangential velocities at the solid surfaces (i.e., outer edges of the double layers) of a stationary circular cylinder and a nearby plane wall moving with a velocity equal to $-v_{\infty} \mathbf{e}_{y}$, which satisfies the boundary conditions

$$
\begin{aligned}
& x=0: \quad \mathbf{v}_{2}=\frac{\varepsilon \zeta_{\mathrm{w}}}{\eta} \nabla \Phi-v_{\infty} \mathbf{e}_{y}, \\
& \psi=\psi_{0}: \quad \mathbf{v}_{2}=\frac{\varepsilon \zeta_{\mathrm{p}}}{\eta} \nabla \Phi \\
& \left(x^{2}+y^{2}\right)^{1 / 2} \rightarrow \infty \quad \text { and } \quad x \geqslant 0: \quad \mathbf{v}_{2} \rightarrow \mathbf{0} .
\end{aligned}
$$

Here the electric potential distribution $\Phi$ is provided by Eq. (25). Superposing the velocity field $\mathbf{v}_{2}$ with $\mathbf{v}_{1}$ yields the total fluid velocity field produced by the transverse electrophoretic motion of a circular cylinder subject to an imposed electric field parallel to a plane wall and specified by Eqs. (26a)-(26c). By obtaining the drag force $\mathbf{F}_{2}$ and torque $\mathbf{T}_{2}$ per unit length exerted by the fluid on the stationary cylinder, individually adding these to the force $\mathbf{F}_{1}$ and torque $\mathbf{T}_{1}$ given by Eqs. (14a) and (14b) with $U_{y}-v_{\infty}$ to replace $U_{y}$, and equating the results to zero, the translational and angular velocities of the cylinder will result.

The stream function $\Psi_{2}$ associated with $\mathbf{v}_{2}$ can also be expressed by Eq. (17), and the coefficients $A, B, C, \ldots$, etc., should be determined by applying Eqs. (27a)-(27c) to Eq. (17) and using Eqs. (12a), (12b) and (25). The procedure is straightforward but tedious, and the result is given in Appendix B. The force $\mathbf{F}_{2}$ and torque $\mathbf{T}_{2}$ per unit length exerted on the stationary cylinder by the fluid due to the electrokinetic motion can be easily obtained by the substitution of the coefficients $A, C$, and $D$ given by Eqs. (B.1), (B.3), and (B.4) into Eqs. (18a), (18b).

\subsection{Derivation of the particle velocities}

Using the constraints that the net force $\mathbf{F}_{1}+\mathbf{F}_{2}$ and net torque $\mathbf{T}_{1}+\mathbf{T}_{2}$ acting on the electrophoretic cylinder must vanish, we obtain the translational and rotational velocities of the cylinder near an insulating plane wall as

$$
U_{x}=\frac{\varepsilon E_{\infty}}{\eta} \tanh \psi_{0}\left[D_{y} \operatorname{sech} \psi_{0}+2 Q_{x y}\left(P_{1} \sinh ^{2} \psi_{0}-1\right)\right],
$$




$$
\begin{aligned}
U_{y}= & \frac{\varepsilon E_{\infty}}{\eta}\left\{\left(M-\zeta_{\mathrm{w}}\right) \operatorname{coth} 2 \psi_{0}-\frac{1}{2} D_{x} \operatorname{csch} \psi_{0}\right. \\
& +\frac{1}{4} Q_{y y}\left[\left(\cosh 4 \psi_{0}-5\right) \operatorname{csch} 2 \psi_{0}\right. \\
& \left.\left.+\sinh 2 \psi_{0}\left(\left(\cosh 2 \psi_{0}-2\right) O_{0}+R_{0}\right)\right]\right\}, \\
\Omega= & \frac{\varepsilon E_{\infty}}{2 \eta a} \operatorname{csch} \psi_{0}\left\{\operatorname { s e c h } ^ { 2 } \psi _ { 0 } \left[-\left(M-\zeta_{\mathrm{w}}\right)\right.\right. \\
& \left.+\frac{1}{2} D_{x}\left(\cosh \psi_{0}+\cosh 3 \psi_{0}\right)\right]+Q_{y y}\left[1+\tanh ^{2} \psi_{0}\right. \\
& \left.\left.+\sinh ^{2} \psi_{0}\left(2+\left(\cosh 2 \psi_{0}-2\right) O_{0}+R_{0}\right)\right]\right\} .
\end{aligned}
$$

Here, $O_{n}, P_{n}$, and $R_{n}$ are defined by Eqs. (B.21)-(B.25). Again, in the limit $\lambda \rightarrow 0$, Eqs. (28a)-(28c) with $\zeta_{\mathrm{w}}=0$ reduces to Eqs. (21a) and (21b) for the electrophoresis of the circular cylinder in an unbounded fluid.

For a uniformly charged circular cylinder undergoing electrophoretic motion in a transversely applied electric field parallel to an insulating plane wall, Eqs. (28a)-(28c) become

$U_{y}=\frac{\varepsilon E_{\infty}}{\eta}\left(\zeta_{\mathrm{p}}-\zeta_{\mathrm{w}}\right) \operatorname{coth} 2 \psi_{0}$,

$\Omega=-\frac{\varepsilon E_{\infty}}{\eta a}\left(\zeta_{\mathrm{p}}-\zeta_{\mathrm{w}}\right) \frac{\operatorname{sech} \psi_{0}}{\sinh 2 \psi_{0}}$,

and $U_{x}=0$. Equations (29a) and (29b) are identical to the previous result obtained by Keh et al. [29].

\subsection{Results and discussion}

The analytical solution for the translational and angular velocities of the circular cylinder undergoing transverse electrophoresis under the applied electric field $\mathbf{E}_{\infty}=E_{\infty} \mathbf{e}_{y}$ parallel to a dielectric plane wall is obtained in Eqs. (28a)-(28c). Again, we consider the four cases of the zeta potential distribution on the surface of the cylindrical particle defined by Eqs. (23a)(23d) as illustrative examples. After calculating the multipole moments according to Eqs. (16a)-(16c) and substituting them into Eqs. (28a)-(28c), we obtain the translational and angular velocities of the particle as functions of the parameter $\lambda$ defined by Eq. (3) for each of the four cases. For convenience in the following discussion, the condition $\zeta_{\mathrm{w}}=0$ will be taken for the plane wall in all cases.

For Case I defined by Eq. (23a), there is no translation in the direction of the applied electric field $\left(U_{y}=0\right)$ and no rotation $(\Omega=0)$ of the cylindrical particle due to the antisymmetry of the zeta potential distribution on the particle surface about the $x$ axis (or the plane $y=0$ ), irrespective of the value of $\lambda$. The result of the lateral velocity of the particle, $U_{x}$, as a function of $\lambda$ is plotted in Fig. 7. The existence of the insulating plane wall enhances the local electric field at the particle surface on the near side to the plane wall in comparison with that on the far side [29], and thus generates a finite lateral velocity of the cylinder in the direction of $\mathbf{e}_{x} \zeta_{0} /\left|\zeta_{0}\right|$ (predictable from observing the strength and direction distributions of the tangential electroosmotic velocity at the particle surface given in

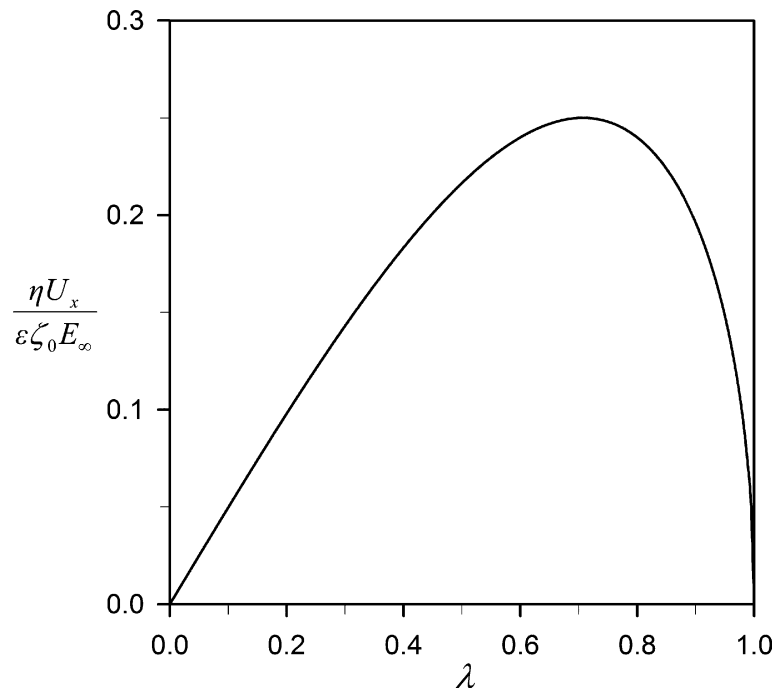

Fig. 7. Plots of the normalized velocity $\eta U_{x} / \varepsilon \zeta_{0} E_{\infty}$ of a circular cylinder with a zeta potential distribution given by Eq. (23a) in a transversely applied electric field parallel to a conducting plane wall versus the separation parameter $\lambda$.

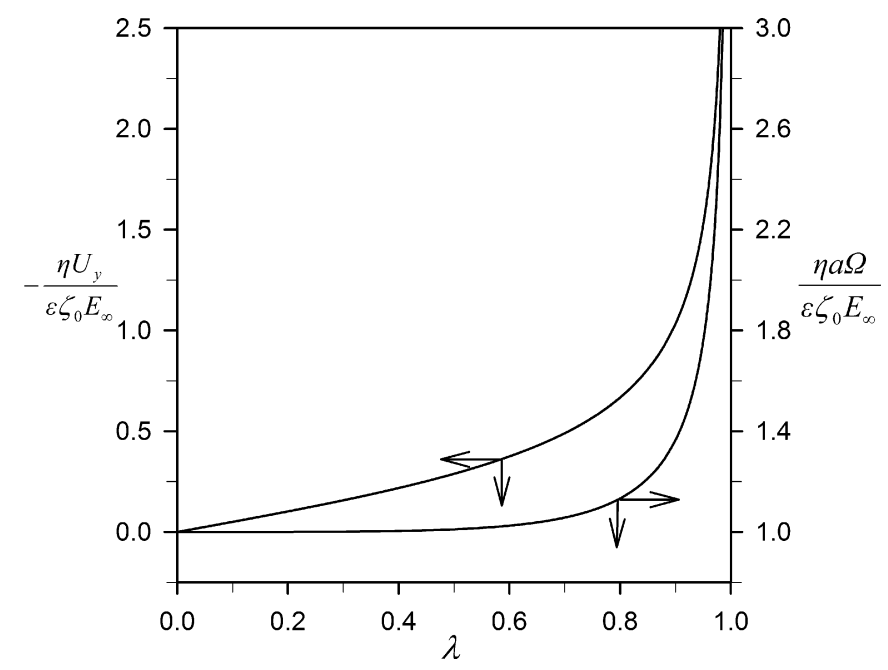

Fig. 8. Plots of the normalized velocities $-\eta U_{y} / \varepsilon \zeta_{0} E_{\infty}$ and $\eta a \Omega / \varepsilon \zeta_{0} E_{\infty}$ of a circular cylinder with a zeta potential distribution given by Eq. (23b) in a transversely applied electric field parallel to a conducting plane wall versus the separation parameter $\lambda$.

Eq. (26b)) as long as $0<\lambda<1$, but this velocity disappears in both limits of $\lambda$. It can be found that the maximal magnitude of $U_{x}$ equals $\varepsilon\left|\zeta_{0}\right| E_{\infty} / 4 \eta$, which occurs at $\lambda=1 / \sqrt{2}$.

For Case II, the lateral velocity $U_{x}=0$ for any value of $\lambda$ owing to the symmetry of the zeta potential distribution on the surface of the cylindrical particle about the $x$ axis. The results of the translational velocity $U_{y}$ in the direction of the imposed electric field and of the angular velocity $\Omega$ of the particle as functions of $\lambda$ are plotted in Fig. 8. For an isolated cylinder (with $\lambda=0$ ), the particle rotates with an angular velocity $\Omega=\varepsilon \zeta_{0} E_{\infty} / \eta a$ without translation, as given by Eqs. (21a) and (21b). The existence of the dielectric plane wall (with a finite value of $\lambda$ ) increases this angular velocity and causes a finite translational velocity $U_{y}$ of the cylinder in the direction of $-\mathbf{e}_{y} \zeta_{0} /\left|\zeta_{0}\right|$. Both magnitudes of $\Omega$ and $U_{y}$ increase with an 


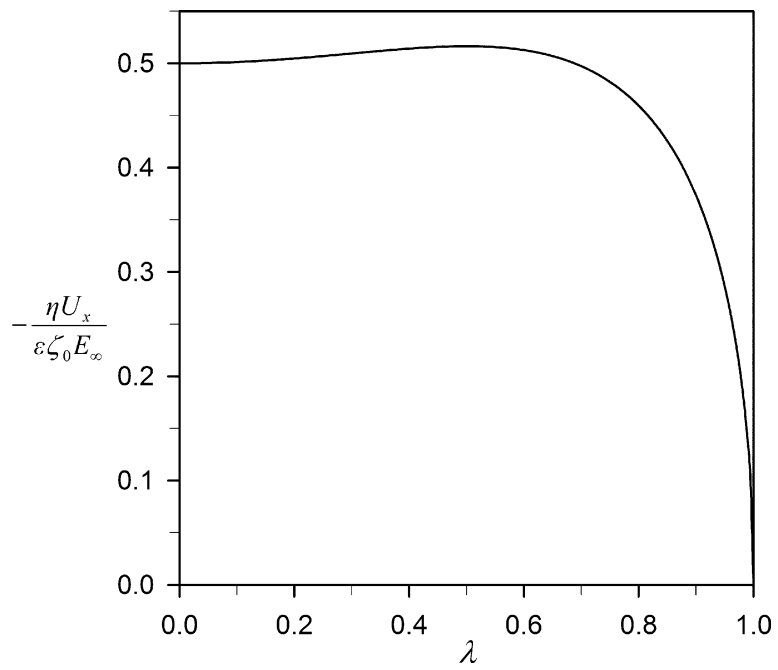

Fig. 9. Plots of the normalized velocity $-\eta U_{x} / \varepsilon \zeta_{0} E_{\infty}$ of a circular cylinder with a zeta potential distribution given by Eq. (23c) in a transversely applied electric field parallel to a conducting plane wall versus the separation parameter $\lambda$.

increase in the parameter $\lambda$ to infinity in the limit $\lambda=1$ (in which the local tangential electric field at the contact point becomes infinity). This behavior is predictable from observing the strength and direction distributions of the tangential electroosmotic velocity at the particle surface given in Eq. (26b).

For Case III defined by Eq. (23c), $U_{y}=\Omega=0$ due to the antisymmetry of the zeta potential distribution on the particle surface about the $x$ axis, regardless of the value of $\lambda$. The result of the lateral velocity $U_{x}$ of the particle as a function of $\lambda$ is plotted in Fig. 9. For the case of an isolated cylinder (with $\lambda=0$ ), the particle translates with a lateral velocity $U_{x}=-\varepsilon \zeta_{0} E_{\infty} / 2 \eta$ as predicted by Eq. (21a). The approach of an insulating plane wall (with an increase in $\lambda$ ) first increases the magnitude of this lateral velocity of the cylinder (the influence of the enhancement of the local electric field at the particle surface is stronger than the effect of the viscous retardation caused by the wall) to a maximum at a finite value of $\lambda$, then reduces it (the effect of the viscous retardation dominates) to zero in the limit $\lambda=1$. The maximal magnitude of $U_{x}$ equals about $0.516 \varepsilon\left|\zeta_{0}\right| E_{\infty} / \eta$, which takes place near $\lambda=0.500$.

For Case IV, there is no velocity of the circular cylinder in the direction perpendicular to the applied electric field $\left(U_{x}=0\right)$ for any value of $\lambda$, owing to the symmetry of the zeta potential distribution on the particle surface about the $x$ axis. The results of the electrophoretic velocities $U_{y}$ and $\Omega$ of the particle as functions of $\lambda$ are plotted in Fig. 10. For an isolated cylinder (with $\lambda=0$ ), the particle translates with a velocity $U_{y}=\varepsilon \zeta_{0} E_{\infty} / 2 \eta$ without rotation, as given by Eqs. (21a) and (21b). The existence of a nearby insulating plane wall (with a finite value of $\lambda$ ) increases this electrophoretic velocity and causes a finite angular velocity $\Omega$ of the cylinder in the direction of $-\mathbf{e}_{z} \zeta_{0} /\left|\zeta_{0}\right|$. Both magnitudes of $U_{y}$ and $\Omega$ increase with an increase in the parameter $\lambda$ to infinity in the limit $\lambda=1$ (where the local tangential electric field at the contact point is infinity).

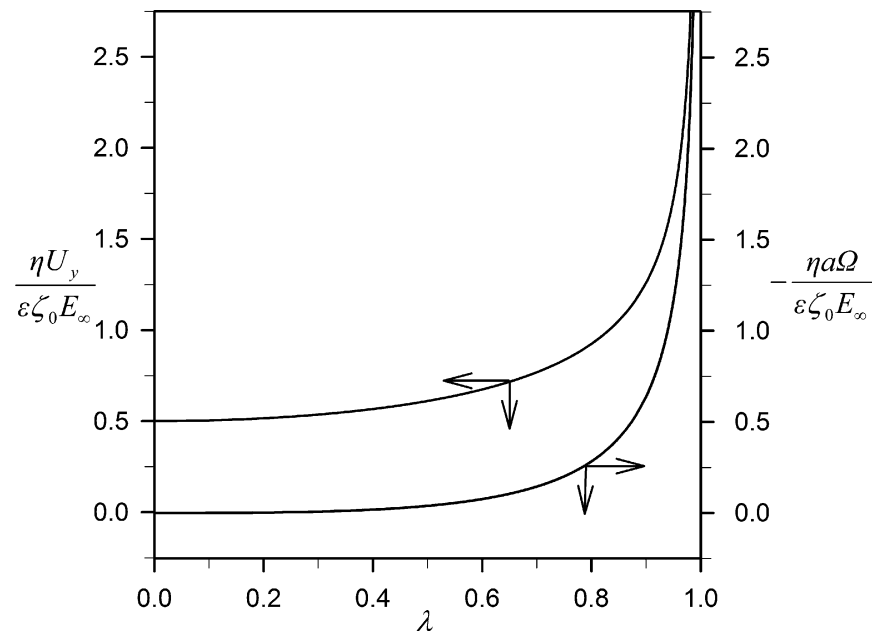

Fig. 10. Plots of the normalized velocities $\eta U_{y} / \varepsilon \zeta_{0} E_{\infty}$ and $-\eta a \Omega / \varepsilon \zeta_{0} E_{\infty}$ of a circular cylinder with a zeta potential distribution given by Eq. (23d) in a transversely applied electric field parallel to a conducting plane wall versus the separation parameter $\lambda$.

\section{Concluding remarks}

In this work, the two-dimensional (transverse) electrophoretic motion of a dielectric circular cylinder with a general angular zeta potential distribution on its surface in the proximity of a large plane wall has been analytically investigated at the quasisteady state. The thickness of the electric double layers adjacent to the solid surfaces is assumed to be much smaller than the particle radius and the gap width between the solid surfaces. A cylindrical bipolar coordinate system has been used to solve the Laplace and Stokes equations for the electric potential and velocity fields, respectively, in the fluid phase in two fundamental cases: external electric fields applied normal to a conducting plane wall and parallel to a dielectric plane wall. The translational and angular velocities of the cylindrical particle are obtained in explicit expressions (20a)-(20c) and (28a)-(28c) for the two cases. Before using these equations, one has only to evaluate the multipole moments of the zeta potential distribution at the particle surface defined by Eqs. (15) and (16a)-(16c). The contributions from the electroosmotic flow produced by the interaction of the tangentially applied electric field with the thin electric double layer adjacent to the plane wall and from the wall-corrected electrophoretic driving force to the particle velocities can be superimposed due to the linearity of the problem. Several illustrative examples of the cylindrical particle-plane wall system with odd and even zeta potential distributions, respectively, are given to discuss in detail the boundary effects on the electrophoretic velocities of the nonuniformly charged particle.

For the purpose of obtaining analytical solutions for the transverse electrophoretic motion of a circular cylinder in the proximity of a plane wall parallel to its axis, the effect of the ends of the cylinder has been ignored in our analyses. In order to investigate the electrophoresis of a cylinder having a relatively large but finite length with or without a confining plane wall, the use of a slender-body theory $[42,57]$ or numerical calculations might be needed. However, our results in Eqs. (20a)-(20c) and 
(28a)-(28c) demonstrate that the electrophoretic velocities of the cylinder are independent of its length (or the size of the particle). Therefore, it is reasonable to expect that, unless the zeta potential or surface charge density at the ends of the cylinder is relatively high, the end effect on its electrophoretic velocities will not be significant.

\section{Acknowledgment}

This research was partly supported by the National Science Council of the Republic of China.

\section{Appendix A. Coefficients in Eq. (17) satisfying boundary conditions given by Eq. (13) with $U_{x}=U_{y}=\Omega=0$}

In Section 2, the coefficients in Eq. (17) for the stream function determined using the boundary conditions given by Eq. (13) with $U_{x}=U_{y}=\Omega=0$ and Eqs. (9), (12), and (15) are obtained as follows:

$$
\begin{aligned}
A= & D_{y}\left(1+2 \psi_{0} \operatorname{coth} \psi_{0}\right) \operatorname{sech} 2 \psi_{0} \frac{\cosh \psi_{0}}{\psi_{0}} \\
& -Q_{y x} \frac{\operatorname{csch} 4 \psi_{0}}{2 \psi_{0}}\left\{16 \psi_{0}+5 \sinh 2 \psi_{0}+\sinh 6 \psi_{0}\right. \\
& -8 \cosh ^{2} \psi_{0}\left[2 \psi_{0}+\cosh 2 \psi_{0}\left(P_{0} \sinh \psi_{0}\right.\right. \\
& \left.\left.\left.\times\left(4 \psi_{0}+\sinh 2 \psi_{0}\right)-4 \psi_{0}\right)\right]\right\},
\end{aligned}
$$

$B=-A$

$$
\begin{aligned}
C= & \frac{\operatorname{sech} 2 \psi_{0}}{\psi_{0}}\left\{-D_{y} \sinh \psi_{0}+\frac{1}{4} Q_{y x} \tanh \psi_{0}\left[6+2 \cosh 4 \psi_{0}\right.\right. \\
& \left.\left.-P_{0}\left(\sinh 3 \psi_{0}+\sinh 5 \psi_{0}\right)\right]\right\},
\end{aligned}
$$

$$
D=\frac{\sinh \psi_{0} \operatorname{sech} 2 \psi_{0}}{\psi_{0} \operatorname{coth} \psi_{0}-1}\left\{2 M \sinh \psi_{0}-D_{x} \tanh \psi_{0}\right.
$$$$
+\frac{1}{4} Q_{x x}\left[\left(\cosh 4 \psi_{0}+1\right) O_{1}+\cosh 2 \psi_{0}\left(R_{1}-2 O_{1}\right)\right.
$$$$
\left.\left.+6 \sinh \psi_{0}-2 \sinh 3 \psi_{0}\right]\right\} \text {, }
$$

$a_{1}=-\frac{2 \psi_{0} \tanh \psi_{0} C+\left(\sinh 2 \psi_{0}-2 \tanh \psi_{0}\right) A}{2\left(\cosh 2 \psi_{0}-1\right)}$,

$b_{1}=\frac{1}{2} A$,

$c_{1}=-a_{1}$,

$a_{n}=\frac{n \sinh \psi_{0} \cosh n \psi_{0}-\cosh \psi_{0} \sinh n \psi_{0}}{\left[n^{2}\left(\cosh 2 \psi_{0}-1\right)+1-\cosh 2 n \psi_{0}\right] \sinh \psi_{0}} H_{n}$ $(n \geqslant 2)$,

$b_{n}=a_{n} \frac{(1-n)\left[\cosh (n-1) \psi_{0}-\cosh (n+1) \psi_{0}\right]}{(1-n) \sinh (n+1) \psi_{0}+(1+n) \sinh (n-1) \psi_{0}}$

$(n \geqslant 2)$,

$c_{n}=-a_{n} \quad(n \geqslant 2)$,

$d_{n}=\frac{1+n}{1-n} b_{n} \quad(n \geqslant 2)$,

$a_{1}^{\prime}=\frac{2 \psi_{0}-\sinh 2 \psi_{0}}{2\left(\cosh 2 \psi_{0}-1\right)} D$, $b_{1}^{\prime}=\frac{1}{2} D$,

$c_{1}^{\prime}=-a_{1}^{\prime}$

$a_{n}^{\prime}=\frac{n \sinh \psi_{0} \cosh n \psi_{0}-\cosh \psi_{0} \sinh n \psi_{0}}{\left[n^{2}\left(\cosh 2 \psi_{0}-1\right)+1-\cosh 2 n \psi_{0}\right] \sinh \psi_{0}} H_{n}^{\prime}$

$(n \geqslant 2)$,

$b_{n}^{\prime}=a_{n}^{\prime} \frac{(1-n)\left[\cosh (n-1) \psi_{0}-\cosh (n+1) \psi_{0}\right]}{(1-n) \sinh (n+1) \psi_{0}+(1+n) \sinh (n-1) \psi_{0}}$

$(n \geqslant 2)$,

$c_{n}^{\prime}=-a_{n}^{\prime} \quad(n \geqslant 2)$,

$d_{n}^{\prime}=\frac{1+n}{1-n} b_{n}^{\prime} \quad(n \geqslant 2)$.

In the above equations,

$$
\begin{aligned}
H_{n}= & \sinh \psi_{0}\left[\left(D_{y}-2 Q_{x y} \cosh \psi_{0}\right)\left(F_{n+1}-F_{n-1}\right)\right. \\
& \left.+2 Q_{x y} \sinh \psi_{0} P_{n}\right] \\
H_{n}^{\prime}= & \frac{1}{2}\left\{-\left[2\left(M-D_{x} \cosh \psi_{0}\right)+\left(1+\cosh 2 \psi_{0}\right) Q_{x x}\right]\right. \\
& \times\left(F_{n+1}+F_{n-1}\right)+\left[4\left(M \cosh \psi_{0}-D_{x}\right)\right. \\
& \left.+\left(5 \cosh \psi_{0}-\cosh 3 \psi_{0}\right) Q_{x x}\right] F_{n} \\
& \left.+Q_{x x}\left[\sinh \psi_{0}\left(R_{n}-5 O_{n}\right)+\sinh 3 \psi_{0} O_{n}\right]\right\},
\end{aligned}
$$

$O_{n}=\sum_{k=1}^{n} F_{k} s_{k n}+\sum_{k=n+1}^{\infty} F_{k} s_{n k}$

$P_{0}=\sum_{k=1}^{\infty}\left(F_{k+1}-F_{k-1}\right) e^{-k \psi_{0}}+F_{1}$

$P_{n}=\sum_{k=1}^{n}\left(F_{k+1}-F_{k-1}\right) c_{k n}+\sum_{k=n+1}^{\infty}\left(F_{k+1}-F_{k-1}\right) c_{n k}+c_{0 k} F_{1}$ $(n \geqslant 1)$,

$R_{n}=\sum_{k=1}^{n}\left(F_{k+2}-F_{k-2}\right) s_{k n}+\sum_{k=n+1}^{\infty}\left(F_{k+2}-F_{k-2}\right) s_{n k}$ $-\left(F_{1}+F_{-1}\right) s_{1 n}$,

$F_{n}=n e^{-n \psi_{0}}\left(\tanh \psi_{0}+1\right)$,

$c_{n k}=2 e^{-k \psi_{0}} \cosh n \psi_{0}$,

$s_{n k}=2 e^{-k \psi_{0}} \sinh n \psi_{0}$.

\section{Appendix B. Coefficients in Eq. (17) satisfying boundary conditions given by Eqs. (27a)-(27c)}

In Section 3, the coefficients in Eq. (17) for the stream function determined using the boundary conditions given by Eqs. (27a)-(27b) with Eqs. (12a), (12b), (15), and (25) are ob- 
tained as follows:

$$
\begin{aligned}
A= & \frac{1}{8 \psi_{0}}\left\{\operatorname { c s c h } { } ^ { 3 } \psi _ { 0 } \left[M \sec h \psi_{0}\left(4 \psi_{0}-\sinh 4 \psi_{0}\right)\right.\right. \\
& \left.+2 D_{x}\left(\sinh 2 \psi_{0}-2 \psi_{0} \cosh 2 \psi_{0}\right)\right] \\
& -2 Q_{y y}\left[2 \left(1+\cosh 2 \psi_{0}-\operatorname{csch}{ }^{2} \psi_{0}\right.\right. \\
& \left.+\psi_{0}\left(\operatorname{coth} \psi_{0}\left(5+\csc h^{2} \psi_{0}\right)-\tanh \psi_{0}\right)\right) \\
& +\operatorname{coth} \psi_{0}\left(4 \psi_{0}+\sinh 2 \psi_{0}\right) \\
& \left.\left.\times\left(2\left(\cosh 2 \psi_{0}-2\right) O_{0}+R_{0}\right)\right]\right\}
\end{aligned}
$$

$B=-A$,

$C=\frac{1}{2 \psi_{0}}\left(2 M \operatorname{coth} 2 \psi_{0}-D_{x} \operatorname{csch} \psi_{0}\right)$

$+\frac{Q_{y y}}{4 \psi_{0}}\left\{2 \tanh \psi_{0}-2 \operatorname{coth} \psi_{0}\right.$

$\left.+\sinh 2 \psi_{0}\left[2+R_{0}+\left(\cosh 2 \psi_{0}-2\right) O_{0}\right]\right\}$,

$$
\begin{aligned}
D= & \frac{1}{2\left(\psi_{0} \operatorname{coth} \psi_{0}-1\right)} \\
& \times\left[D_{y} \operatorname{sech} \psi_{0}+2 Q_{x y}\left(\sinh ^{2} \psi_{0} P_{1}-1\right)\right],
\end{aligned}
$$

$a_{1}=-\frac{2 \psi_{0} \tanh \psi_{0} C+\left(\sinh 2 \psi_{0}-2 \tanh \psi_{0}\right) A}{2\left(\cosh 2 \psi_{0}-1\right)}$,

$b_{1}=\frac{1}{2} A$,

$c_{1}=-a_{1}$,

$a_{n}=\frac{n \sinh \psi_{0} \cosh n \psi_{0}-\cosh \psi_{0} \sinh n \psi_{0}}{\left[n^{2}\left(\cosh 2 \psi_{0}-1\right)+1-\cosh 2 n \psi_{0}\right] \sinh \psi_{0}} L_{n}$

$(n \geqslant 2)$,

$b_{n}=a_{n} \frac{(1-n)\left[\cosh (n-1) \psi_{0}-\cosh (n+1) \psi_{0}\right]}{(1-n) \sinh (n+1) \psi_{0}+(1+n) \sinh (n-1) \psi_{0}}$

$(n \geqslant 2)$,

$c_{n}=-a_{n} \quad(n \geqslant 2)$,

$d_{n}=\frac{1+n}{1-n} b_{n} \quad(n \geqslant 2)$,

$a_{1}^{\prime}=\frac{2 \psi_{0}-\sinh 2 \psi_{0}}{2\left(\cosh 2 \psi_{0}-1\right)} D$,

$b_{1}^{\prime}=\frac{1}{2} D$,

$c_{1}^{\prime}=-a_{1}^{\prime}$

$a_{n}^{\prime}=\frac{n \sinh \psi_{0} \cosh n \psi_{0}-\cosh \psi_{0} \sinh n \psi_{0}}{\left[n^{2}\left(\cosh 2 \psi_{0}-1\right)+1-\cosh 2 n \psi_{0}\right] \sinh \psi_{0}} L_{n}^{\prime}$

$$
(n \geqslant 2) \text {, }
$$

$b_{n}^{\prime}=a_{n}^{\prime} \frac{(1-n)\left[\cosh (n-1) \psi_{0}-\cosh (n+1) \psi_{0}\right]}{(1-n) \sinh (n+1) \psi_{0}+(1+n) \sinh (n-1) \psi_{0}}$ $(n \geqslant 2)$,

$c_{n}^{\prime}=-a_{n}^{\prime} \quad(n \geqslant 2)$,

$d_{n}^{\prime}=\frac{1+n}{1-n} b_{n}^{\prime} \quad(n \geqslant 2)$.
In the above equations,

$$
\begin{aligned}
& L_{n}=\frac{1}{2}\left\{\left[2\left(-M+\cosh \psi_{0} D_{x}\right)+\left(1+\cosh 2 \psi_{0}\right) Q_{y y}\right]\right. \\
& \times\left(G_{n+1}+G_{n-1}\right)+\left[4\left(M \cosh \psi_{0}-D_{x}\right)\right. \\
& \left.+\left(\cosh 3 \psi_{0}-5 \cosh \psi_{0}\right) Q_{y y}\right] G_{n} \\
& \left.-Q_{y y}\left[\sinh \psi_{0}\left(R_{n}-5 O_{n}\right)+\sinh 3 \psi_{0} O_{n}\right]\right\} \text {, } \\
& L_{n}^{\prime}=\sinh \psi_{0}\left[\left(D_{y}-2 Q_{y x} \cosh \psi_{0}\right)\left(G_{n-1}-G_{n+1}\right)\right. \\
& \left.+2 Q_{y x} \sinh \psi_{0} P_{n}\right] \text {, } \\
& O_{0}=\sum_{k=1}^{\infty} G_{k} e^{-k \psi_{0}} \\
& O_{n}=\sum_{k=1}^{n} G_{k} c_{k n}+\sum_{k=n+1}^{\infty} G_{k} c_{n k} \quad(n \geqslant 1) \\
& P_{n}=\sum_{k=1}^{n}\left(G_{k-1}-F_{k+1}\right) s_{k n}+\sum_{k=n+1}^{\infty}\left(G_{k-1}-G_{k+1}\right) s_{n k} \text {, } \\
& R_{0}=\sum_{k=1}^{\infty}\left(G_{k+2}+G_{k-2}\right) e^{-k \psi_{0}}+\left(G_{1}-G_{-1}\right) e^{-\psi_{0}}+G_{2} \text {, } \\
& R_{n}=\sum_{k=1}^{n}\left(G_{k+2}+G_{k-2}\right) c_{k n}+\sum_{k=n+1}^{\infty}\left(G_{k+2}+G_{k-2}\right) c_{n k} \\
& +\left(G_{1}-G_{-1}\right) c_{1 n}+G_{2} c_{0 n} \quad(n \geqslant 1),
\end{aligned}
$$

where $c_{n k}$ and $s_{n k}$ are given by Eqs. (A.26) and (A.27).

\section{References}

[1] M.V. Smoluchowski, Bull. Int. Acad. Sci. Cracovie 8 (1903) 182.

[2] F.A. Morrison, J. Colloid Interface Sci. 34 (1970) 210.

[3] J.L. Anderson, Annu. Rev. Fluid Mech. 21 (1989) 61.

[4] D.C. Henry, Proc. R. Soc. London Ser. A 133 (1931) 106.

[5] R.W. O'Brien, L.R. White, J. Chem. Soc. Faraday Trans. 274 (1978) 1607.

[6] R.W. O’Brien, J. Colloid Interface Sci. 92 (1983) 204.

[7] H.J. Keh, S.B. Chen, Langmuir 9 (1993) 1142.

[8] H.J. Keh, T.Y. Huang, J. Colloid Interface Sci. 160 (1993) 354.

[9] R.W. DeBlois, C.P. Bean, Rev. Sci. Instrum. 41 (1970) 909.

[10] A.G. Ewing, R.A. Wallingford, T.M. Olefirowicz, Anal. Chem. 61 (1989) 292A.

[11] S. Haber, J. Colloid Interface Sci. 179 (1996) 380.

[12] H.J. Keh, J.L. Anderson, J. Fluid Mech. 153 (1985) 417.

[13] F.A. Morrison, J.J. Stukel, J. Colloid Interface Sci. 33 (1970) 88.

[14] H.J. Keh, L.C. Lien, J. Chin. Inst. Chem. Eng. 20 (1989) 283.

[15] M. Loewenberg, R.H. Davis, J. Fluid Mech. 288 (1995) 103.

[16] H.J. Keh, S.B. Chen, J. Fluid Mech. 194 (1988) 377.

[17] E. Yariv, H. Brenner, J. Fluid Mech. 484 (2003) 85.

[18] H.J. Keh, L.C. Lien, J. Fluid Mech. 224 (1991) 305.

[19] H.J. Keh, J.Y. Chiou, AIChE J. 42 (1996) 1397.

[20] E. Yariv, H. Brenner, Phys. Fluids 14 (2002) 3354.

[21] E. Yariv, H. Brenner, SIAM J. Appl. Math. 64 (2003) 423.

[22] Y. Hao, S. Haber, Int. J. Multiphase Flow 24 (1998) 793.

[23] H.N. Unni, H.J. Keh, C. Yang, Electrophoresis 28 (2007) 658.

[24] P.Y. Chen, H.J. Keh, J. Colloid Interface Sci. 286 (2005) 774.

[25] A.L. Zydney, J. Colloid Interface Sci. 169 (1995) 476.

[26] P. Venema, J. Fluid Mech. 282 (1995) 45.

[27] H.J. Keh, J.S. Jan, J. Colloid Interface Sci. 183 (1996) 458.

[28] J. Ennis, J.L. Anderson, J. Colloid Interface Sci. 185 (1997) 497. 
[29] H.J. Keh, K.D. Horng, J. Kuo, J. Fluid Mech. 231 (1991) 211.

[30] J.J. Feng, W.Y. Wu, J. Fluid Mech. 264 (1994) 41.

[31] H.J. Keh, J.H. Hsu, J. Colloid Interface Sci. 221 (2000) 210.

[32] M.Z. Bazant, T.M. Squires, Phys. Rev. Lett. 92 (2004) 066101.

[33] S.J. Miklavic, D.Y.C. Chan, L.R. White, T.W. Healy, J. Phys. Chem. 98 (1994) 9022.

[34] M.L. Grant, D.A. Saville, J. Colloid Interface Sci. 171 (1995) 35.

[35] J.L. Anderson, J. Colloid Interface Sci. 105 (1985) 45.

[36] M. Teubner, J. Phys. Chem. 76 (1982) 5564.

[37] B.J. Yoon, J. Colloid Interface Sci. 142 (1991) 575

[38] Y.E. Solomentsev, Y. Pawar, J.L. Anderson, J. Colloid Interface Sci. 158 (1993) 1.

[39] D. Velegol, J.D. Feick, L.R. Collins, J. Colloid Interface Sci. 230 (2000) 114.

[40] J.Y. Kim, B.J. Yoon, J. Colloid Interface Sci. 262 (2003) 101.

[41] M.C. Fair, J.L. Anderson, J. Colloid Interface Sci. 127 (1989) 388.

[42] Y. Solomentsev, J.L. Anderson, J. Fluid Mech. 279 (1994) 197.

[43] D. Long, A. Ajdari, Phys. Rev. Lett. 81 (1998) 1529.

[44] J.D. Feick, D. Velegol, Langmuir 16 (2000) 10315.
[45] J.Y. Kim, B.J. Yoon, J. Colloid Interface Sci. 251 (2002) 318.

[46] J.D. Feick, D. Velegol, Langmuir 18 (2002) 3454.

[47] J.D. Feick, N. Chukwumah, A.E. Noel, D. Velegol, Langmuir 20 (2004) 3090.

[48] G.P. Crawford, IEEE Spectrum 37 (10) (2000) 40.

[49] J.M. Crowley, N.K. Sheridon, L. Romano, J. Electrostat. 55 (2002) 247.

[50] C.F. Edman, R.B. Swint, C. Gurtner, R.E. Formosa, S.D. Roh, K.E. Lee, P.D. Swanson, D.E. Ackley, J.J. Coleman, M. Heller, J. IEEE Photonics Technol. Lett. 12 (2000) 1198.

[51] P.A. Smith, C.D. Nordquist, T.N. Jackson, T.S. Mayer, B.R. Martin, J. Mbindyo, T.E. Mallouk, Appl. Phys. Lett. 77 (2000) 1399.

[52] H.J. Keh, T.H. Hsieh, Langmuir 23 (2007) 7928.

[53] G.B. Jeffery, Proc. R. Soc. London A 101 (1922) 169.

[54] J. Happel, H. Brenner, Low Reynolds Number Hydrodynamics, Nijhoff, Dordrecht, 1983.

[55] H. Helmholtz, Ann. Phys. (Leipzig) 7 (1879) 337.

[56] S. Wakiya, J. Phys. Soc. Jpn. 39 (1975) 1603.

[57] R.G. Cox, J. Fluid Mech. 44 (1970) 791. 